\title{
Using IACTs to Measure the Profiles of Muons in TeV Air Showers
}

\section{A. M. W. Mitchell*}

University of Zurich, Zurich, Switzerland

E-mail: alison.mitchellephysik.uzh.ch

\section{H. P. Dembinski}

Max Planck Institute for Nuclear Physics, Heidelberg, Germany

E-mail: hans.dembinski@mpi-hd.mpg.de

\section{R. D. Parsons}

Max Planck Institute for Nuclear Physics, Heidelberg, Germany

E-mail: daniel.parsons@mpi-hd.mpg.de

\begin{abstract}
Muons are regularly detected by IACTs as part of normal observations, producing distinctive ringshaped images that are routinely used for calibration. Due to the stereoscopic trigger requirement of IACT arrays, often both the muon ring and associated air shower are contained in the same event. We explore the potential of reconstructing $\mathrm{TeV}$ air showers associated with detectable muons, in order to measure the effective muon density and to reconstruct the lateral and longitudinal muon profiles. We optimise cuts and study the acceptance of IACTs to muons, exploring also the muon purity and identification efficiency. Although detection is feasible, the acceptance with a H.E.S.S.-like telescope array is found to be limited. The application to CTA with a dedicated reconstruction algorithm for muons and their associated air showers is promising. ${ }^{\dagger}$
\end{abstract}

36th International Cosmic Ray Conference -ICRC2019-

July 24th - August 1st, 2019

Madison, WI, U.S.A.

\footnotetext{
* Speaker.

${ }^{\dagger}$ The study presented in these proceedings is published in [1].
} 


\section{Introduction}

In high energy Extensive Air Showers (EAS), the number of measured muons in EAS with primary energy $\gtrsim 10^{17} \mathrm{eV}$ is typically in excess of the number predicted by the most recent hadronic interaction models, despite the inclusion of LHC data. The situation deteriorates towards higher energies, with the discrepancy being recently confirmed at $8 \sigma$ in a combined statistical analysis of the available data [2].

Imaging Atmospheric Cherenkov Telescopes (IACTs) are sensitive to sub-PeV EAS; an energy regime where the true cosmic ray composition is known from satellite measurements and the primary energy is within coverage of the LHC fixed target equivalent energy $10^{17} \mathrm{eV}$. Recent studies have shown, however, that there remain significant differences between hadronic interaction models in the predicted muon flux for sub-PeV showers with $100 \mathrm{GeV}$ primary energy [3]. Therefore, an IACT measurement of muon properties in TeV EAS may provide useful input towards resolving whether the measured discrepancies originate in the extrapolation from LHC data to higher energies (in case of agreement between simulations and data), or to more fundamental properties of EAS development in the case of a disagreement.
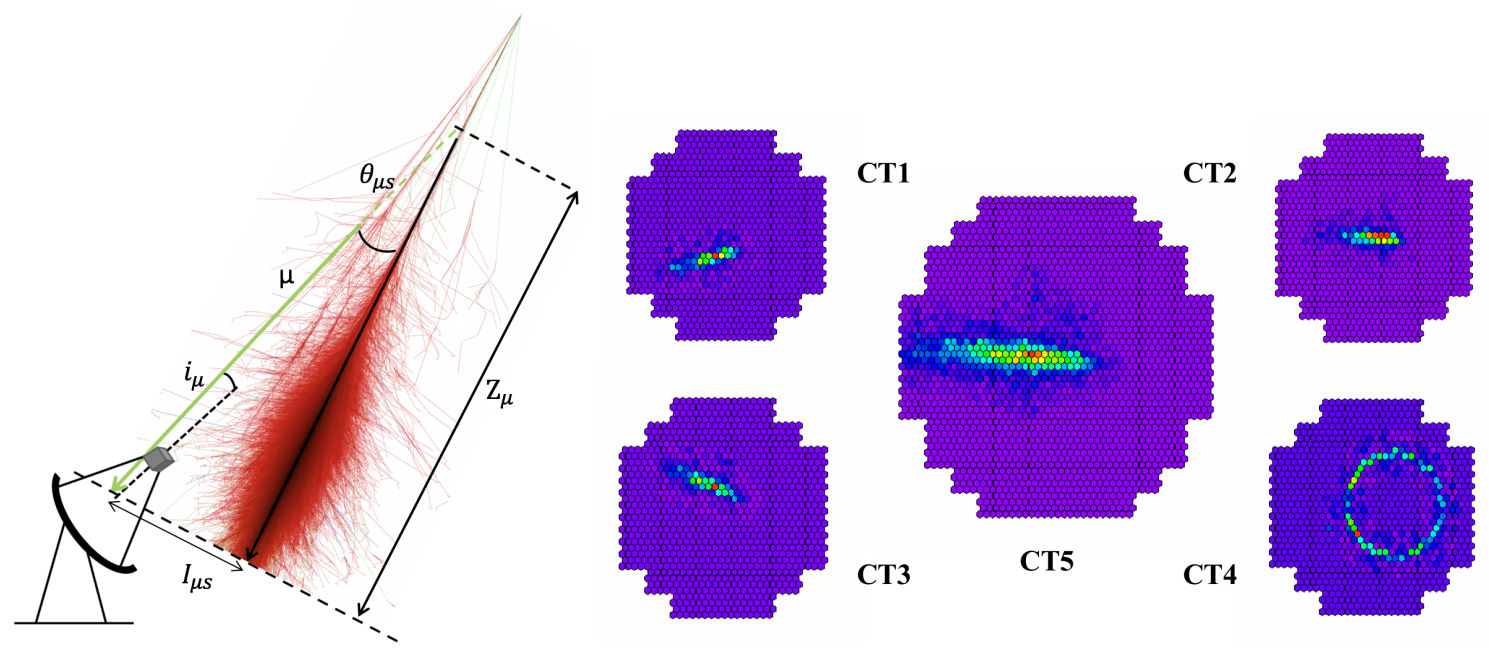

Figure 1: A $10 \mathrm{TeV}$ proton shower event. Left: geometrical schematic of a muon arriving in an IACT at an angle $\theta_{\mu s}$ to the parent shower, originating from a production slant height $Z_{\mu}$ (see main text for more details, shower image taken from [4]). Right: images of a $10 \mathrm{TeV}$ proton shower event as seen by HESS, with a muon contained in CT4.

H.E.S.S. (High Energy Stereoscopic System) is an array of five IACTs located in the Khomas Highlands of Namibia, with four $105 \mathrm{~m}^{2}$ mirror area telescopes operational since 2004, arranged in a square with $100 \mathrm{~m}$ sides and a fifth central $612 \mathrm{~m}^{2}$ mirror area telescope added to the array in $2012[7,8]$.

For the purposes of this study, proton and iron showers with primary energies from $2-150 \mathrm{TeV}$ at the H.E.S.S. array were simulated with an $E^{-2}$ spectrum using the Corsika package. An additional set of monoenergetic $10 \mathrm{TeV}$ primary energy proton and iron simulations with full particle tracking enabled were used to investigate the IACT acceptance to muons. Table 1 summarises the 


\begin{tabular}{llcl} 
Model & Primary & Energy Range & $\mathrm{N}$ Showers \\
\hline QGSJetII-04 & proton & $10 \mathrm{TeV}$ & $1 \times 10^{6}$ \\
QGSJetII-04 & iron & $10 \mathrm{TeV}$ & $8 \times 10^{5}$ \\
EPOS LHC & proton & $10 \mathrm{TeV}$ & $2 \times 10^{4}$ \\
QGSJetII-04 & proton & $0.8-150 \mathrm{TeV}$ & $1.2 \times 10^{7}$ \\
QGSJetII-04 & iron & $2-150 \mathrm{TeV}$ & $5 \times 10^{6}$ \\
EPOS LHC & proton & $0.8-150 \mathrm{TeV}$ & $1.2 \times 10^{7}$ \\
EPOS LHC & iron & $2-150 \mathrm{TeV}$ & $5 \times 10^{6}$
\end{tabular}

Table 1: CORSIKA simulations used in this study. For the simulations over an energy range, an $E^{-2}$ spectrum was assumed and events were re-weighted in the analysis to reproduce the CR spectrum. The monoenergetic simulations and a small sub-sample of the spectrum simulations included full particle tracking to ground level.

simulation statistics; two post-LHC hadronic interaction models (QGSJetII-04 and EPOS LHC) were used $[5,6]$.

\section{Muon Acceptance and Shower Reconstruction}

The IACT muon identification efficiency (fraction of muons hitting a telescope that are identified) improves with increasing distance between the shower core axis and the muon direction, $I_{\mu s}$, as the muon image becomes less contaminated by the parent shower (see Figure 2). Muon identification efficiency also improves with decreasing angular distance, $i_{\mu}$, to the telescope optical axis; the more on-axis the muon, the more complete the ring image formed.
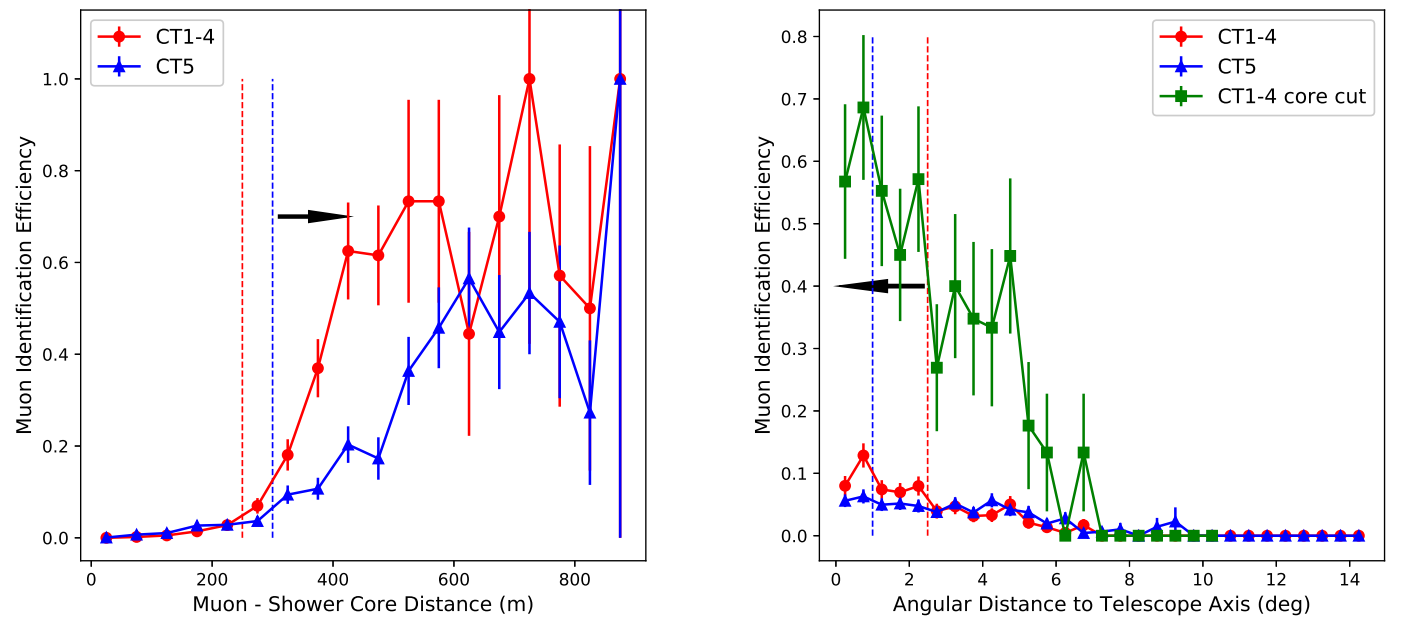

Figure 2: Muon identification efficiency for CT1-4 and CT5. Left: as a function of distance between the muon and the shower core. Right: as a function of angular distance from the telescope axis. The muon identification efficiency is much improved for muons with a minimum distance from the shower core reducing the image contamination (green points). 


\begin{tabular}{lcc} 
Variable & Loose & Tight \\
\hline Neighbouring Pixels & $\langle N\rangle<4$ & $\langle\boldsymbol{N}\rangle<\mathbf{3 . 5}$ \\
Ring Completeness & $30 \%$ & $\mathbf{6 0 \%}$ \\
Ring Width & $\mathbf{0 . 0 2}^{\circ}-\mathbf{0 . 2}$ & $0.04^{\circ}-0.08^{\circ}$ \\
Impact Parameter & $\mathbf{0 - 1 2} \mathbf{~ m}$ & $0.9-6.5 \mathrm{~m}$ \\
Ring Radius & $\mathbf{0 . 9}^{\circ}-\mathbf{1 . 5}^{\circ}$ & $1.0^{\circ}-1.5^{\circ}$ \\
Outer Ring Radius & $<\mathbf{3 . 5 ^ { \circ }}$ & $<2.2^{\circ}$
\end{tabular}

Table 2: Selected cut parameters for identifying muon events within H.E.S.S., quoted for CT1-4. The final set of cuts adopted are indicated in bold (see also Figure 3). Cuts for CT5 differ slightly in the impact parameter and outer ring radius (ring radius + muon ring centre offset from camera centre) [10].

Muons are traditionally used almost exclusively for calibration purposes by IACTs, for which muon purity (fraction of events identified as muons that are true muons) is paramount. For measurements of physical properties, however, it is important to optimise the trade-off between muon identification efficiency and muon purity.

The intrinsic acceptance of IACTs to muons as a function of $i_{\mu}$ and $I_{\mu s}$, using the ring-fitting algorithm of [9] (as for the standard procedure for H.E.S.S. calibration with muons [10]), results in a muon purity of $\sim 20 \%$ and an identification efficiency of $\sim 45 \%$ for loose cuts. This is shown in figure 3 - further improvements in muon purity can be made by adjusting the cut parameters; however, this tends to come at a cost of reduced identification efficiency.

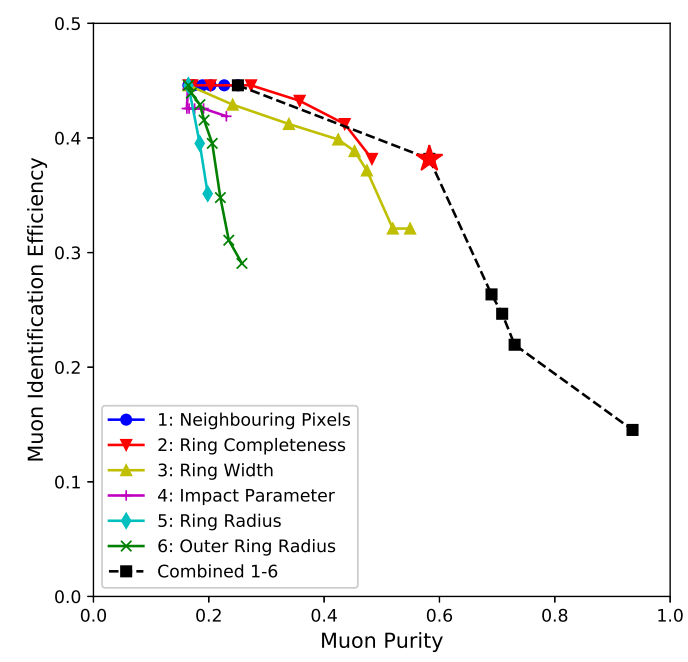

Figure 3: The effect of tightening cut parameters tends to improve muon purity and decrease muon identification efficiency. An optimised cut combination (red star) of Neighbouring Pixels and Ring Completeness only, is found by combining cuts in order at their tightest values, with all other cuts remaining loose (see also Table 2).

Cut variables used as listed in table 2 include the average number of neighbouring pixels (after image cleaning), the 'Ring Completeness' (percentage of full ring covered by non-zero pixels), the 
distance of the muon impact position from the centre of the telescope mirror ('Impact Parameter') and the furthest edge of the muon ring from the camera centre (i.e. ring radius + offset of muon ring centre from camera centre), 'Outer Ring Radius'.

Further improvements to both the muon identification efficiency and muon purity are likely possible with alternative muon identification algorithms, cut parameter optimisation or additional cut parameters, enabling for example the identification of partial muon rings in images otherwise dominated by the parent shower.

\section{Measurable Properties of Muons in EAS}

Here we consider three properties of muons in EAS of interest to hadronic interaction models and CR composition - the muon content, longitudinal distribution and lateral distribution.

\subsection{Muon event rate}

Figure 4 shows that the muon content is higher in iron showers than for proton showers with the same primary energy - a systematic shift of 15\% more muons was also seen in QGSJetII-04 than in EPOS LHC. However, the ratio between the number of muons in proton, $N_{\mu}^{p}$ and iron initiated EAS is larger (at up to $\sim 10$ ) than the expected relation:

$$
N_{\mu}=A^{1-\beta} N_{\mu}^{p}
$$

where $\beta \simeq 0.9$ is a constant and for iron with $A \approx 56$, a factor $\sim 1.4$ more muons are expected for the same shower energy [11]. There is presumably a strong selection effect towards improved muon identification in iron EAS due to the increased number of sub-showers and muons scattering further from the shower core, as well as an intrinsically higher muon content. The muon event rate is therefore a strongly detector dependent quantity and difficult to compare between experiments.

\subsection{Muon production point: slant height}

The longitudinal distribution (slant height, $Z_{\mu}$ ) corresponds to the muon production point along the shower axis. This is reconstructed from the muon track (given the muon impact position and direction) and the shower core axis (given the shower core position and direction). The extrapolated intersection point, assuming that the muon originated from the shower axis and using the height corresponding to the point of closest approach, gives the slant height. This can be seen to underestimate slightly the true height, likely a consequence of neglecting the shower breadth due to the transverse momentum provided by the parent pions and kaons prior to their decay into muons.

\subsection{Muon lateral distribution}

The muon lateral distribution, a measure of the muon density $\rho_{\mu}$ as a function of distance to the shower core $r$, is defined by:

$$
\rho_{\mu}=\frac{N_{\mu}}{2 \pi r \mathrm{~d} r N_{\mathrm{ev}}},
$$

where $\mathrm{d} r$ is the radial width of the bin at a distance $r$ from the shower core; $N_{\mu}$ is the number of muons and $N_{\mathrm{ev}}$ is the total number of events in each radial bin. The reconstruction is performed 

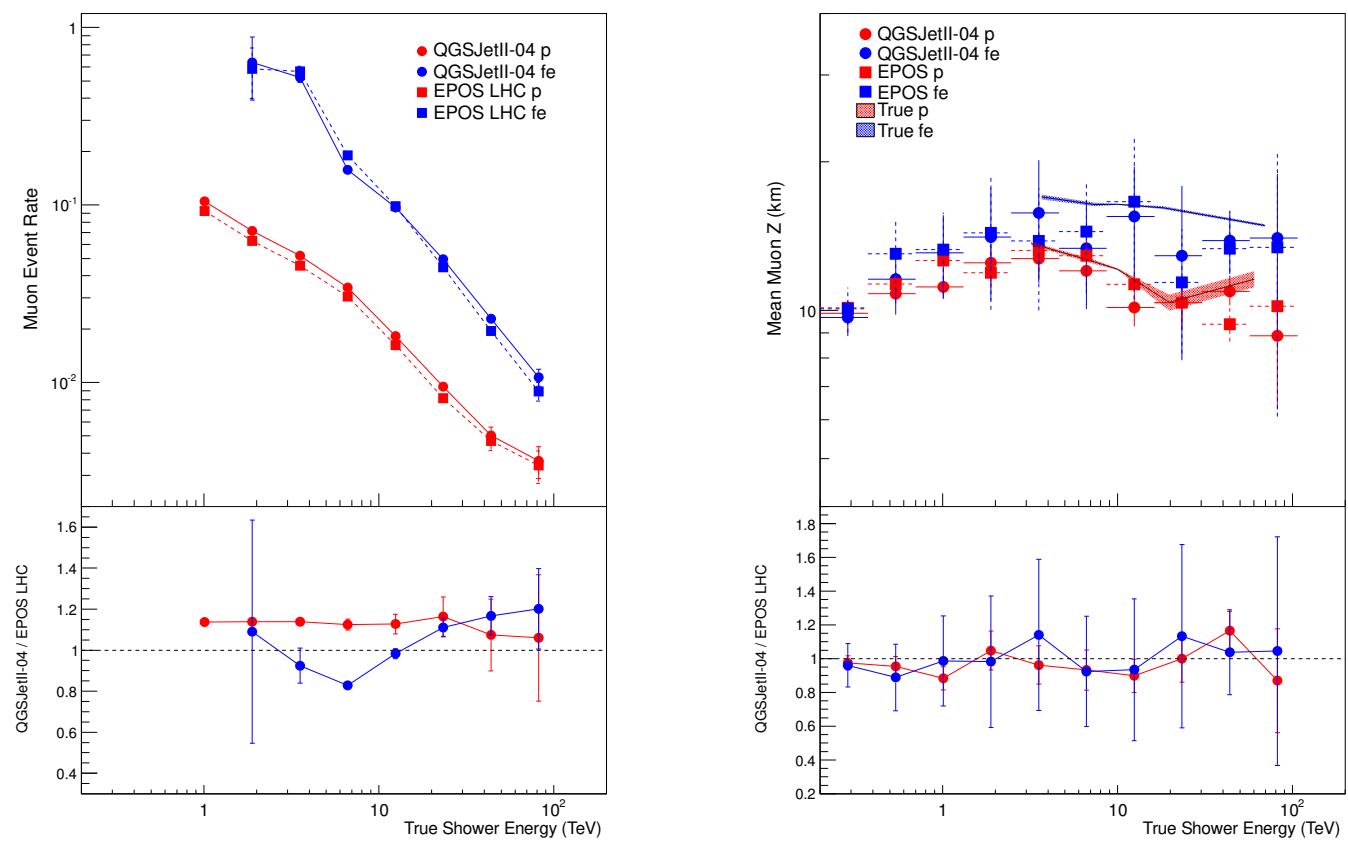

Figure 4: Left: Muon event rate from proton and iron showers with true shower energy. Right: The reconstructed muon slant height $\mathrm{Z}_{\mu}$ was found to be consistent with true production height values.
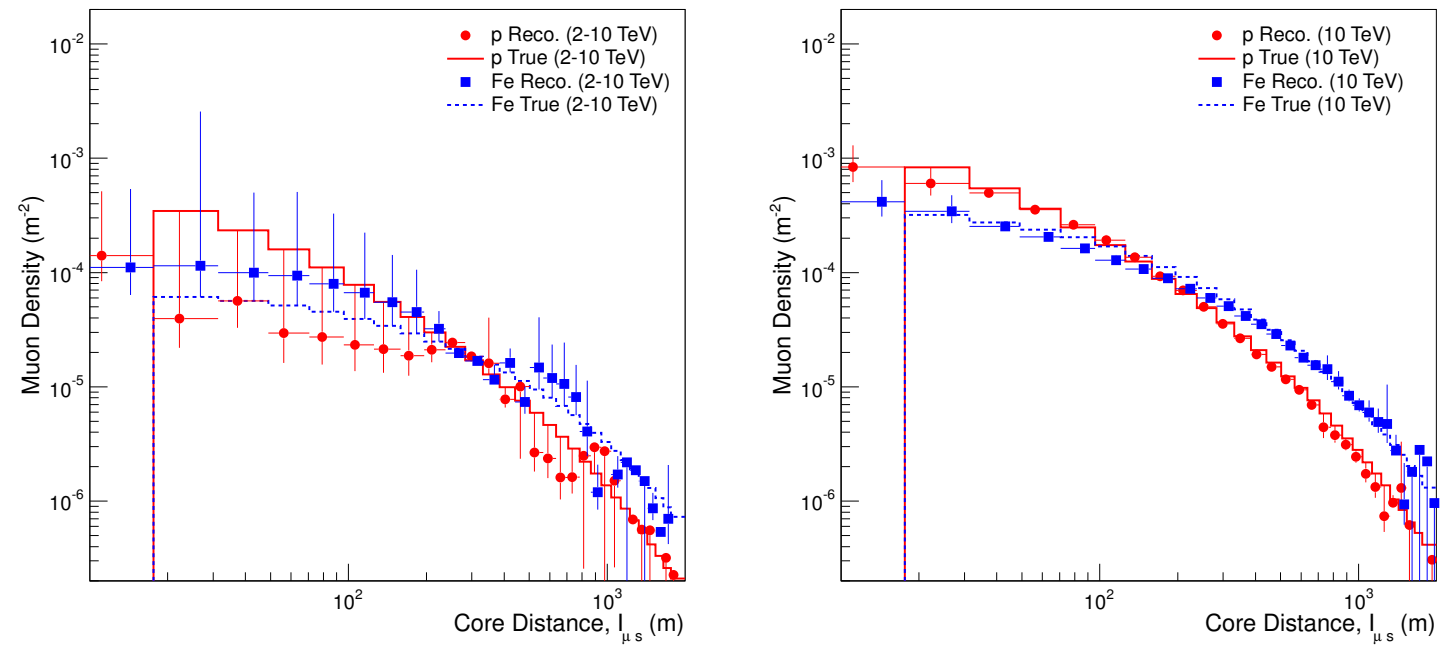

Figure 5: Muon lateral distribution as reconstructed in the shower plane using simulations of HESS (left) and a toy model for CTA (right). The reconstruction is performed using an effective area to a mixed proton and iron primary composition, error bars are statistical showing the most pessimistic uncertainties of the method. Shown for QGSJetII-04 only. 
in the tilted shower plane, such that a zenith angle dependent factor of $\cos \theta_{z}$ is already taken into account.

In order to reconstruct the measured muon lateral distribution, an effective area $A_{\text {eff }}$ is found using the measured muon density $\rho_{\mu}^{*}$ from simulations including acceptance effects:

$$
\rho_{\mu}^{*}=\frac{N_{\mu}^{*}}{A_{\mathrm{eff}} N_{\mathrm{ev}}^{*}},
$$

where $N_{\mu}^{*}$ and $N_{\mathrm{ev}}^{*}$ are the number of events triggering at least two telescopes and number of muons detected in each radial bin. Therefore, the reconstructed lateral distribution (3.3) would equal the true (3.2) by definition when using this approach on simulations. However, given the limited capabilities of IACTs to identify proton and iron initiated showers unambiguously, the muon lateral distribution was constructed using an effective area for a mixed composition of 50\% proton and $50 \%$ iron. The error bars in figure 5 indicate the extreme values given by the effective areas for pure proton and pure iron samples (i.e. reconstructing the muon lateral distribution for protons with $A_{\text {eff }}$ for pure protons agrees by definition, whereas using $A_{\text {eff }}$ for pure iron is maximally pessimistic). It can be seen that there is some separation power available within a limited core distance range in figure 5 (left).

Using the same simulations a toy model for the expected performance of the Cherenkov Telescope Array (CTA) was constructed assuming that the acceptance of the CTA medium and large telescopes to muons is analogous to the different telescope sizes of HESS, with the acceptance ratio scaling similarly between the small and medium CTA telescopes as for the medium to large [12].

The muon lateral distribution was reconstructed in this toy model using same approach as outlined above - the performance is markedly improved with respect to that of the HESS experiment (figure 5, right). As the same simulations were used, this is likely due to improved shower sampling by the increased telescope coverage of CTA, as well as the assumption of a pure muon sample after the identification efficiencies found using the projected acceptance behaviour. The shower core resolution was assumed to be reconstructed to a comparable accuracy to that achieved with HESS.

Several effects likely to degrade the performance with respect to this expectation were omitted; sample contamination, cuts based on the number of triggered telescopes, image selection cuts and the poor energy resolution of hadronic showers. However, competing effects which will likely improve the performance were also neglected, including the increased field-of-view. signal integration window and lower trigger threshold of CTA telescopes, as well as improvements in muon identification algorithms. When performing this analysis on data it will be necessary to use fixed zenith angle and energy bins. Some energy resolution of the hadronic EAS is therefore also required - the energy resolution capabilities were not investigated in this study, using instead the true energy as known from the MC sample.

\section{Conclusions and Outlook}

There are several options to improve the performance of current generation experiments above that presented here. Refining algorithms for muon identification; enabling muons to be identified even at low ring completeness such as by use of a Hough transform or image analysis techniques; would improve the muon identification efficiency. Some of the biases inherent in this analysis 
would be reduced; in particular, the ratio of muon content between proton and iron showers is much larger than expected, likely due to a strong identification efficiency bias, as muons in proton showers are likely to remain closer to the shower core, more contaminated by Cherenkov emission from the parent shower and hence harder to identify.

A first measurement of these parameters describing muons in TeV EAS by current generation IACT arrays would be a welcome input to hadronic interaction models, complementary to previous measurements at higher energies [13, 14]. On data, separation where possible of proton-like and iron-like showers becomes important as the true primary mass is not known apriori. It is challenging to distinguish between proton and iron showers of different energies, as increasing energy often produces similar effects to the global shower development as an increase in primary mass for the same energy. Nevertheless, several options exist, such as the identification of direct Cherenkov light from the primary particle, where the intensity contained in the single pixel is directly proportional to the particle mass. Although the rates of such events are low, given the $\gtrsim 10$ years of data available with current experiments a measurement with increased statistics from that shown here should be possible. Combining direct Cherenkov light with more global shower properties to better identify EAS primary particles and resolve the cosmic ray composition is an approach demonstrated to be feasible with IACTs by [15].

With the forthcoming CTA, an analogous measurement should be possible after accumulating only a few months of data, whilst binned measurements in energy and zenith angle should be possible with five years of data accumulation with the full array [12]. CTA will undoubtedly be able to improve on these measurements and offers powerful capabilities, in physically relevant quantities beyond gamma-rays.

\section{References}

[1] Mitchell, A. M. W., Dembinski, H. P., Parsons, R. D. Astropart. Phys. 111, $23-34$ (2019)

[2] Dembinski, H. P. et. al., proc. UHECR 2018

[3] Parsons, R. D. and Schoorlemmer, H., accepted by Phys. Rev. D arXiv:1904.05135

[4] CORSIKA - an Air Shower Simulation Program, KIT (2017)

[5] Ostapchenko, S., Phys. Rev. D 83, 014018 (2011)

[6] Pierog, T., Karpenko, I., Katzy, J. M., Yatsenko, E. and Werner, K, Phys. Rev. C 92, 034906 (2015)

[7] Aharonian, F. et al., A \& A 457, 899-915 (2006)

[8] Hinton, J. A. New Astr. Rev. 48, 331-337 (2004)

[9] Chaudhuri, B. B. and Kundu, P., Pattern Recognition Letters, 14, 1-6 (1993)

[10] Mitchell, A. M. W., Marandon, V. and Parsons, R. D., proc. 34 ${ }^{\text {th }}$ ICRC (2015)

[11] Dembinski, H. P. Astropart. Phys. 102, 89-94 (2018)

[12] Acharya et. al., Astropart. Phys. 43, 3-18 (2013)

[13] Aab, A. et. al., Phys. Rev. D 91, 032003 (2015)

[14] Apel, W. D. et. al., Astropart. Phys. 34, 476-485 (2011)

[15] Kieda, D. B., Swordy, S. P., Wakely, S. P. Astropart. Phys. 15, 287-303 (2001) 